\title{
Diversity analysis of gut microbiota in osteoporosis and osteopenia patients
}

\author{
Jihan Wang ${ }^{\text {Corresp.. }}{ }^{1}$ ， Yangyang Wang ${ }^{2}$, Wenjie Gao ${ }^{1}$, Biao Wang ${ }^{1}$, Heping Zhao ${ }^{1}$, Yuhong Zeng ${ }^{1}$, Yanhong \\ $\mathbf{J i}^{3}$, Dingjun Hao ${ }^{1}$ \\ ${ }^{1}$ Hong Hui Hospital, Xi'an Jiaotong University, Xi'an, China \\ 2 The Tenth Research Institute of Telecommunications Technology, Xi'an, China \\ 3 Department of Pathogenic Biology and Immunology, Key Laboratory of Environment and Genes Related to Diseases, Ministry of Education of China, \\ School of Basic Medical Sciences, Xi'an Jiaotong University Health Science Center, Xi'an, China \\ Corresponding Author: Jihan Wang \\ Email address: 513837742@qq.com
}

Some evidence suggest that bone health can be regulated by gut microbiota. To better understand this, we performed $16 \mathrm{~S}$ ribosomal RNA sequencing to analyze the intestinal microbial diversity in primary osteoporosis (OP) patients, osteopenia (ON) patients and normal controls (NC). We observed an inverse correlation between the number of bacterial taxa and the value of bone mineral density. The diversity estimators in OP and ON groups were increased compared with that in NC group. Beta diversity analyses based on hierarchical clustering and principal coordinate analysis (PCOA ) could discriminate the NC samples from OP and ON samples. Firmicutes, Bacteroidetes, Proteobacteria and Actinobacteria constituted the four dominant phyla in all samples. Proportion of Firmicutes was significantly higher and Bacteroidetes was significantly lower in OP samples than that in NC samples ( $p<0.05$ ), Gemmatimonadetes and Chloroflexi were significantly different between OP and NC group as well as between ON and NC group $(p<0.01)$. A total of 21 genera with proportions above $1 \%$ were detected and Bacteroides accounted for the largest proportion in all samples. The Blautia, Parabacteroides and Ruminococcaceae genera differed significantly between the OP and NC group $(p<0.05)$. Linear discriminant analysis (LDA) results showed 1 phylum community and 7 phylum communities were enriched in ON and OP, respectively. 35 genus communities, 5 genus communities and 2 genus communities were enriched in OP, ON and NC, respectively. The results of this study indicate that gut microbiota may be a critical factor in osteoporosis development, which can further help us search for novel biomarkers of gut microbiota in OP and understand the interaction between gut microbiota and bone health. 
Jihan Wang ${ }^{\#}{ }^{1}$ Yangyang Wang ${ }^{\#},{ }^{2}$ Wenjie $\mathrm{Gao}^{\#},{ }^{1}$ Biao Wang, ${ }^{1}$ Heping Zhao, ${ }^{1}$ Yuhong Zeng ${ }^{1}$, 3 Yanhong $\mathrm{Ji}^{*},{ }^{3}$ and Dingjun $\mathrm{Hao}^{* 1}$

$4 \quad{ }^{1}$ Hong Hui Hospital, Xi'an Jiaotong University, Xi'an, China

$5 \quad{ }^{2}$ The Tenth Research Institute of Telecommunications Technology, Xi'an, China

$6 \quad{ }^{3}$ Department of Pathogenic Biology and Immunology, Key Laboratory of Environment and

7 Genes Related to Diseases, Ministry of Education of China, School of Basic Medical Sciences,

8 Xi'an Jiaotong University Health Science Center, Xi'an, China

9 \#These authors contributed equally to this work.

10 *Corresponding author: Yanhong Ji, jiyanhong@xjtu.edu.cn; Dingjun Hao, haodingjun@126.com

\section{ABSTRACT}

Some evidence suggest that bone health can be regulated by gut microbiota. To better understand this, we performed $16 \mathrm{~S}$ ribosomal RNA sequencing to analyze the intestinal microbial diversity in primary osteoporosis (OP) patients, osteopenia $(\mathrm{ON})$ patients and normal controls (NC). We observed an inverse correlation between the number of bacterial taxa and the value of bone mineral density. The diversity estimators in $\mathrm{OP}$ and $\mathrm{ON}$ groups were increased compared with that in NC group. Beta diversity analyses based on hierarchical clustering and principal coordinate analysis (PCoA) could discriminate the NC samples from OP and ON samples. Firmicutes, Bacteroidetes, Proteobacteria and Actinobacteria constituted the four dominant phyla in all samples. Proportion of Firmicutes was significantly higher and Bacteroidetes was significantly lower in OP samples than that in NC samples $(p<0.05)$, Gemmatimonadetes and Chloroflexi were significantly different between OP and NC group as well as between ON and NC group $(p<0.01)$. A total of 21 genera with proportions above $1 \%$ were detected and Bacteroides accounted for the largest proportion in all samples. The Blautia, Parabacteroides and Ruminococcaceae genera differed significantly between the OP and NC group $(\mathrm{p}<0.05)$. Linear discriminant analysis (LDA) results showed 1 phylum community and 7 phylum communities were enriched in ON and OP, respectively. 35 genus communities, 5 genus communities and 2 genus communities were enriched in $\mathrm{OP}, \mathrm{ON}$ and $\mathrm{NC}$, respectively. The results of this study indicate that gut microbiota may be a critical factor in osteoporosis development, which can further help us search for novel biomarkers of gut microbiota in OP and understand the interaction between gut microbiota and bone health.

\section{INTRODUCTION}

Osteoporosis is a type of bone-thinning disorder, characterized by a reduction of bone mass, microarchitecture deterioration and an increased risk of fragility fracture. It is the most common reason for a broken bone among the elder. As the population grows and ages, the number of patients with osteoporosis is expected to increase. A decline in bone mineral density (BMD) is the primary cause of fragility fracture ( $\mathrm{Lu}$ et al. 2016). As a metabolic procedure, bone 
41 homeostasis relies on a balance between bone formation (osteoblast-regulated) and bone

42

resorption (osteoclast-regulated) (Chung et al. 2014; Harada \& Rodan 2003). Hereditary characteristics and environmental factors can regulate the complex process of bone metabolism and significantly contribute to age-related bone loss (Pollitzer \& Anderson 1989).

Recently, the gut microbiota have attracted attention in connection with metabolic diseases. The human gastrointestinal tract are colonized by rich and dynamic communities of microbes. The microbes has been considered as a critical factor for metabolic disorders including obesity, diabetes, and osteoporosis (Ejtahed et al. 2016). Therefore, it may represent a novel potential biomarker for the diagnosis and treatment of metabolic disorders (Steves et al. 2016). So far, the effect of gut microbiota on bone health is a relatively new field of research. Several studies have reported it as a regulator of bone mass (McCabe et al. 2015; Sjogren et al. 2012; Weaver 2015) through mediation of the immune system (e.g, osteoclastogenesis), intestinal calcium absorption and the release of neurotransmitters (e.g, serotonin). A better understanding of structure and function changes of microbes will help us search for novel biomarkers and understand the interaction between gut microbiota and bone mass disorder. However, to our knowledge, it remains unclear how gut microbiota changes in osteoporosis patients.

Traditional methods for research on bacterial community inhabitants include isolation, cultivation, and optical microscopy. These approaches are insufficient to obtain relatively fullscale and accurate results about the structure and diversity of microbiota communities in specific samples, because the vast majority of bacteria in fecal samples are anaerobic and cannot be isolated in the laboratory (Perry et al. 2010). High-throughput sequencing has recently been used for bacterial diversity analysis ( $\mathrm{Li}$ et al. 2016a; $\mathrm{Li}$ et al. 2016b). This approach overcomes the limitations of traditional technology and can effectively capture the genomic information of uncultured microorganisms, which may be pathogenic or important for biological processes.

The present study was to explore the bacterial community structure and diversity changes of gut microbiota in patients with primary osteoporosis and primary osteopenia based on 16S rRNA gene sequencing. Results of our research will lay a foundation for searching novel microbe biomarkers and understanding the potential mechanisms of effects of gut microbiota on bone health.

\section{METHODS}

\section{Subject recruitment and bone mineral density detection}

Participants in this study were recruited from Hong Hui Hospital, Xi'an Jiaotong University, Xi'an, China. Dual X-ray absorptiometry (DXA) was performed to detect the bone mineral density of lumbar vertebrae of subjects. We further excluded all patients with any malignancy, chronic liver disease, heart disease, kidney disease, or diabetes. Finally, a total of 18 subjects including six with primary osteoporosis $(\mathrm{OP})$, six with primary osteopenia $(\mathrm{ON})$, and six normal controls (NC; as determined by physical examination) were selected for further research (Table 1). None of the 18 participants ingested yogurt, prebiotics, or probiotics during the fecal collection period, nor had they used medication (e.g., antibiotics) within one month of sample collection. The study was approved by Hong Hui Hospital, Xi'an Jiaotong University, 
82 Biomedical research ethics committee. Each participant provided his or her written informed 83 consent.

84

85

86

87

88

89

90

91

92

93

94

95

96

97

98

\section{Fecal sample collection and DNA extraction}

Fresh stool samples were collected in sterile boxes, then frozen and stored at $-80^{\circ} \mathrm{C}$ for further use. The microbial genome was extracted using QIAamp Fast DNA Stool Mini Kit (Qiagen, Hilden, Germany) according to the manufacturer's instructions. Sample DNA purity and concentration were tested using a Nanodrop 2000 Spectrophotometer.

\section{S rRNA PCR and Illumina sequencing}

We amplified the bacterial 16S ribosomal RNA gene V3-V4 region using the TransGen AP22102 Kit (TransGen, Beijing, China). The following PCR primers were used: 338F 5'ACTCCTACGGGAGGCAGCAG-3' and 806R 5'-GGACTACHVGGGTWTCTAAT-3'. The reaction volume $(20 \mu \mathrm{l})$ comprised $5 \times$ FastPfu Buffer $(4 \mu \mathrm{l}), 2.5 \mathrm{mM}$ dNTPs $(2 \mu \mathrm{l})$, forward primer $(0.8 \mu \mathrm{l}), 5 \mu \mathrm{M}$ reverse primer $(0.8 \mu \mathrm{l})$, FastPfu Polymerase $(0.4 \mu \mathrm{l})$, and template DNA (10 ng). Cycling proceeded as follows: $3 \mathrm{~min}$ at $95^{\circ} \mathrm{C}$; twenty-seven cycles $\left(30 \mathrm{~s}\right.$ at $95^{\circ} \mathrm{C}, 30 \mathrm{~s}$ at $55{ }^{\circ} \mathrm{C}, 45 \mathrm{~s}$ at $\left.72^{\circ} \mathrm{C}\right) ; 10 \mathrm{~min}$ at $72^{\circ} \mathrm{C}$. After amplicons extraction, samples were purified and quantified using the AxyPrep DNA Gel Extraction Kit (Axygen Biosciences, CA, U.S.) and QuantiFluor ${ }^{\text {TM}}$-ST (Promega, U.S.), respectively. Purified amplicons were pooled in equimolar proportions and paired-end sequenced $(2 \times 250 \mathrm{bp})$ on the Illumina MiSeq platform with TruSeqTM DNA Sample Prep Kit (Illumina, U.S.).

\section{S rRNA gene sequencing analysis}

Raw fastq files were demultiplexed, quality-filtered by Trimmomatic and merged by FLASH with the following criteria: (i) The reads were truncated at any site receiving an average quality score $<20$ over a $50 \mathrm{bp}$ sliding window. (ii) Primers were exactly matched allowing 2 nucleotide mismatching, and reads containing ambiguous bases were removed. (iii) Sequences whose overlap longer than $10 \mathrm{bp}$ were merged according to their overlap sequence. Operational taxonomic units (OTUs) were clustered with 97\% similarity cutoff (Edgar 2013) using UPARSE (version $7.1 \mathrm{http} / / /$ drive5.com/uparse/) and chimeric sequences were identified and removed using UCHIME. The taxonomy of each 16S rRNA gene sequence was assigned by QIIME (version 1.7, http://qiime.org/home_static/dataFiles.html) (Caporaso et al. 2010) using RDP Classifier algorithm (http://rdp.cme.msu.edu/) (Wang et al. 2007) against the Silva (SSU123) 16S rRNA database (Quast et al. 2013) using confidence threshold of 70\%. Alpha diversity at the OTU level (e.g., Ace, Chao, Shannon and Simpson index) were calculated in QIIME following previously described methods (Jiang \& Takacs-Vesbach 2017; Lauber et al. 2009; Van Horn et al. 2016). 


\section{Statistical analysis}

Results analysis and figure generation based on clinicopathological information, alpha estimators and relative bacterial abundance were performed using SPSS 21.0 and GraphPad Prism 5.01 software. Student's t-test and the Mann-Whitney U-test were performed, with $p<0.05$ indicating a significant difference between groups. Rarefaction curves were generated based on the alpha diversity estimators. The unweighted UniFrac algorithm was applied for hierarchical clustering and principal coordinates analysis at the OTU level to analyze beta diversity. We applied "Vennerable" package in R software (version 3.3.3) for the generation of venn diagram based at the OTU level. The circos software (http://circos.ca/software/download/circos/) was performed for the generation of collonearity diagram to visualize the corresponding abundance relationship between samples and bacterial communities at the phylum and genus levels. The enriched and significant bacteria in each group were identified by linear discriminant analysis (LDA) combined with effect-size measurements (LEfSe) (Segata et al. 2011), with $p<0.05$. For the Kruskal-Wallis test, LDA values $>2$ were considered significant (Szafranski et al. 2015).

\section{RESULTS}

\section{Illumina sequencing data characteristics}

The clinicopathological information for each of the three groups included in the study is presented in Table 1. There were no significant differences in terms of age or gender, while BMD, T-score and Z-score differed significantly among groups. Illumina sequencing captured a total of 694232 high-quality sequences, with an average of 38568 sequences/sample. Detailed information on the sequence results obtained for each sample are presented in Table S1.

Table 1. Clinicopathological information of the study participants.

\begin{tabular}{lllllll}
\hline Group & Case & Gender & Age & $\begin{array}{l}\text { BMD } \\
\mathrm{L}_{1-4}\left(\mathrm{~g} / \mathrm{cm}^{2}\right)\end{array}$ & Z-score $\mathrm{L}_{1-4}$ & T-score $\mathrm{L}_{1-4}$ \\
\hline $\begin{array}{l}\text { Normal control } \\
\text { (NC) }\end{array}$ & 6 & $\begin{array}{l}\text { Female: } 5 \\
\text { Male: } 1\end{array}$ & $64.80 \pm 5.93$ & $0.81 \pm 0.08$ & $0.12 \pm 0.45$ & $-0.42 \pm 0.26$ \\
$\begin{array}{l}\text { Osteopenia } \\
\text { (ON) }\end{array}$ & 6 & $\begin{array}{l}\text { Female: } 5 \\
\text { Male: } 1\end{array}$ & $67.17 \pm 8.30$ & $0.75 \pm 0.04^{*}$ & $-0.22 \pm 0.50$ & $-2.15 \pm 0.34^{* *}$ \\
$\begin{array}{l}\text { Osteoporosis } \\
\text { (OP) }\end{array}$ & 6 & $\begin{array}{l}\text { Female: } 5 \\
\text { Male: } 1\end{array}$ & $70.00 \pm 7.77$ & $0.61 \pm 0.06^{* * \#}$ & $-1.18 \pm 0.73^{* * \#}$ & $-3.57 \pm 0.46^{* * \#}$ \\
\hline
\end{tabular}

Compares with NC group: ${ }^{*} \mathrm{P}<0.05,{ }^{* *} \mathrm{P}<0.01$. Compares with ON group: ${ }^{\#} \mathrm{P}<0.05,{ }^{\#} \mathrm{P}<0.01$.

$\mathrm{BMD}, \mathrm{Z}$-score and T-score were collected from dual X-ray absorptiometry detection, $\mathrm{L}_{1-4}$ represents lumbar vertebrae 1-4. BMD: bone mineral density. Z-score: the Z-score is the comparison to the age-matched normal. T-score: the T-score is the relevant measure when screening for osteoporosis. The criteria of the World Health Organization are: Normal is a T-score of -1.0 or higher; Osteopenia is defined as between -1.0 and -2.5 ; Osteoporosis is defined as -2.5 or lower.

\section{Inverse correlation between the number of bacterial taxa and the value of BMD}

Based on the sequencing data, the gut microbiota of all samples were classified to 507 OTUs, 
152

153

154

155

156

157

158

367 species, 235 genera, 99 families, 63 orders, 38 classes, 25 phyla. The number of bacterial taxa tended to increase at each level in accordance with the reduction in BMD, as shown in Table 2 and Figure S1. Figure 1 presents a Venn diagram for the OP, ON and NC groups (at the OTU level). There were 455, 378, and 282 OTUs present in the OP, ON, and NC group, respectively. In addition, 208 OTUs (41\%) were shared by all samples; 154 OTUs (30.4\%) were shared between the OP and ON groups. For the remaining components (28.6\%), the OP group (13.6\%) accounted for nearly half of all OTUs.

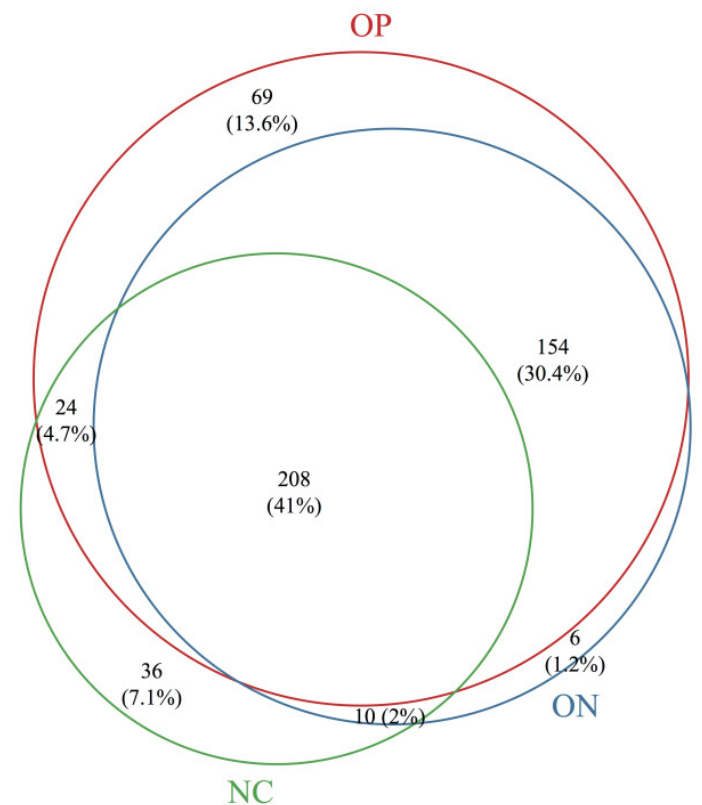

Figure 1. Venn diagram of $\mathrm{OP}, \mathrm{ON}$ and $\mathrm{NC}$ groups at $\mathrm{OTU}$ level.

Table 2. Bacterial taxa in each group at different levels.

\begin{tabular}{llllllll}
\hline & Phylum & Class & Order & Family & Genus & Species & OTU \\
\hline NC & 8 & 14 & 20 & 41 & 134 & 218 & 282 \\
ON & 21 & 33 & 56 & 88 & 195 & 296 & 378 \\
OP & 23 & 35 & 58 & 92 & 219 & 335 & 455 \\
Total & 25 & 38 & 63 & 99 & 235 & 367 & 507 \\
\hline
\end{tabular}

\section{Diversity analysis of gut microbiota in osteoporosis and osteopenia patients}

To determine alpha diversity, we calculated the mean ace index, chao index, shannon index, and simpson reciprocal index. This process allowed us to fully characterize the bacterial community diversity in samples. Detailed information on the estimators in each sample is presented in Table S2. The OTU level rarefaction curves of diversity estimators reached plateau phase (Figure S2), indicating that most bacterial species had been captured by sequencing in all samples. Higher numbers of the estimators represent greater diversity, which suggests that alpha diversity index was inversely correlated with BMD, although there were no significant differences between the OP and ON groups, as shown in Figure 2. 
A

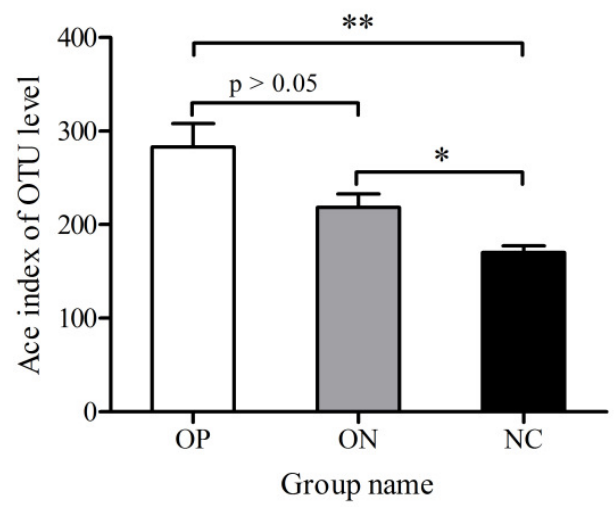

C

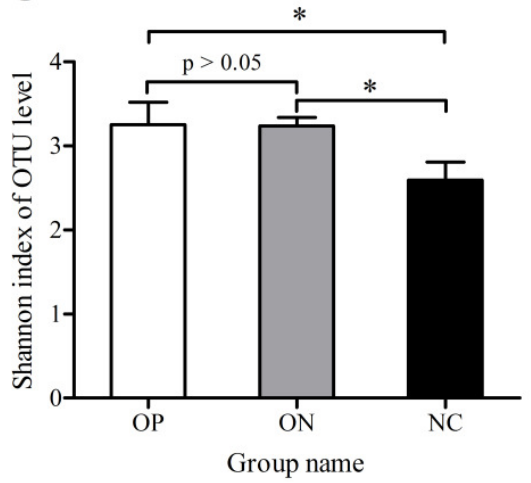

B
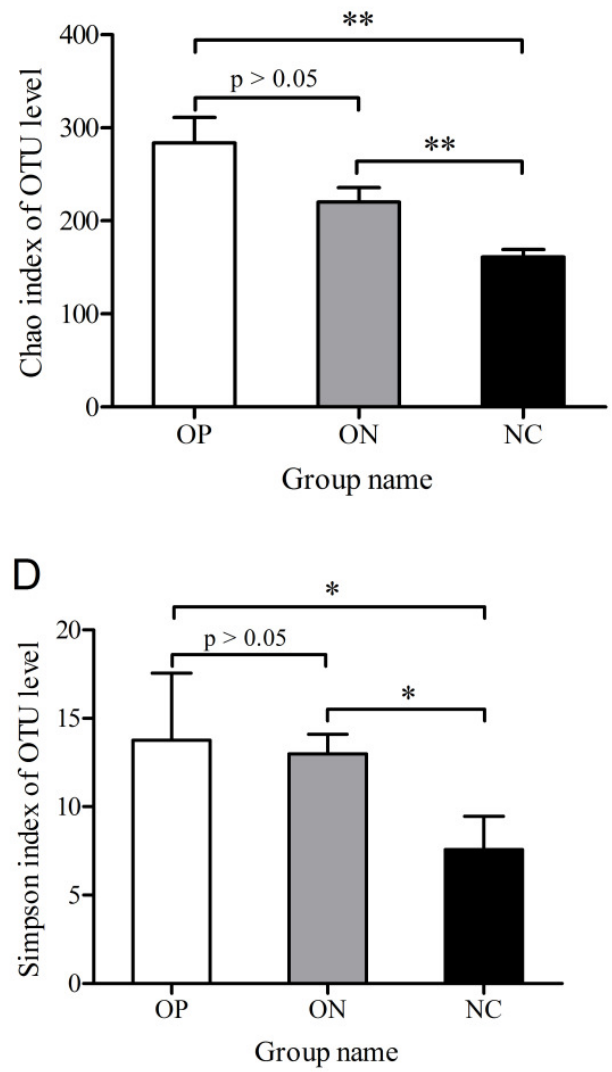

Figure 2. Significance of alpha diversity estimators between different groups. ${ }^{*} 0.01<\mathrm{p} \leq 0.05$,

$$
* * 0.001<\mathrm{p} \leq 0.01 \text {. }
$$

With regard to beta diversity, unweighted UniFrac analysis indicated that hierarchical clustering and principal coordinate analysis ( $\mathrm{PCoA}$ ) could discriminate the $\mathrm{NC}$ samples from $\mathrm{OP}$ as well as ON samples. However, there was substantial overlap between the OP and ON groups, and most ON samples were positioned in the middle of the OP and NC samples, as Figure 3 illustrates.
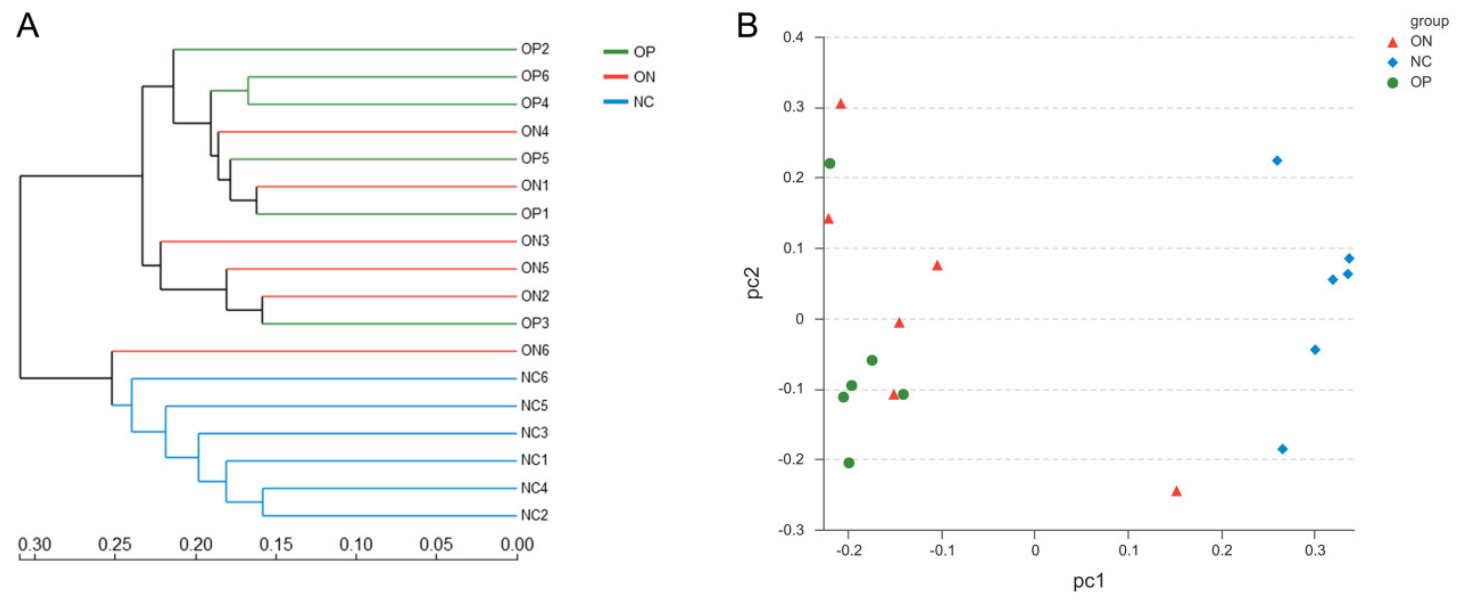

Figure 3. Beta diversity analysis of OP, ON and NC group at OTU level. (A) the hierarchical 
clustering tree. $(B)$ Principal coordinate analysis (PCoA) scatter plot.

\section{Significance analysis of gut bacterial community abundance in osteoporosis and osteopenia patients}

At the phylum level illustrated in Figure 4, Firmicutes, Bacteroidetes, Proteobacteria and Actinobacteria constituted the four dominant phyla in all samples. The average ratios of Firmicutes/Bacteroidetes were 3.326, 1.755 and 1.290 in the OP, ON, and NC groups, respectively. Furthermore, we calculated the significance of the 10 most dominant phyla of microbial community structure among the OP, ON, and NC groups. Differences among the four dominant phyla (Firmicutes, Bacteroidetes, Proteobacteria, and Actinobacteria) were not statistically significant for comparisons between the OP and ON group or the ON and NC group $(p>0.05)$. Proportion of Firmicutes was significantly higher and Bacteroidetes proportion was significantly lower in OP samples than that in the NC group $(\mathrm{p}<0.05)$ (Figure 4B). As for other bacterial communities with small proportions, most of them were rare in the NC group but increased in the OP and ON groups. Gemmatimonadetes and Chloroflexi were significantly different between the OP and NC groups $(p<0.01)$ as well as between the ON and NC groups $(p$ $<0.01)$.

A
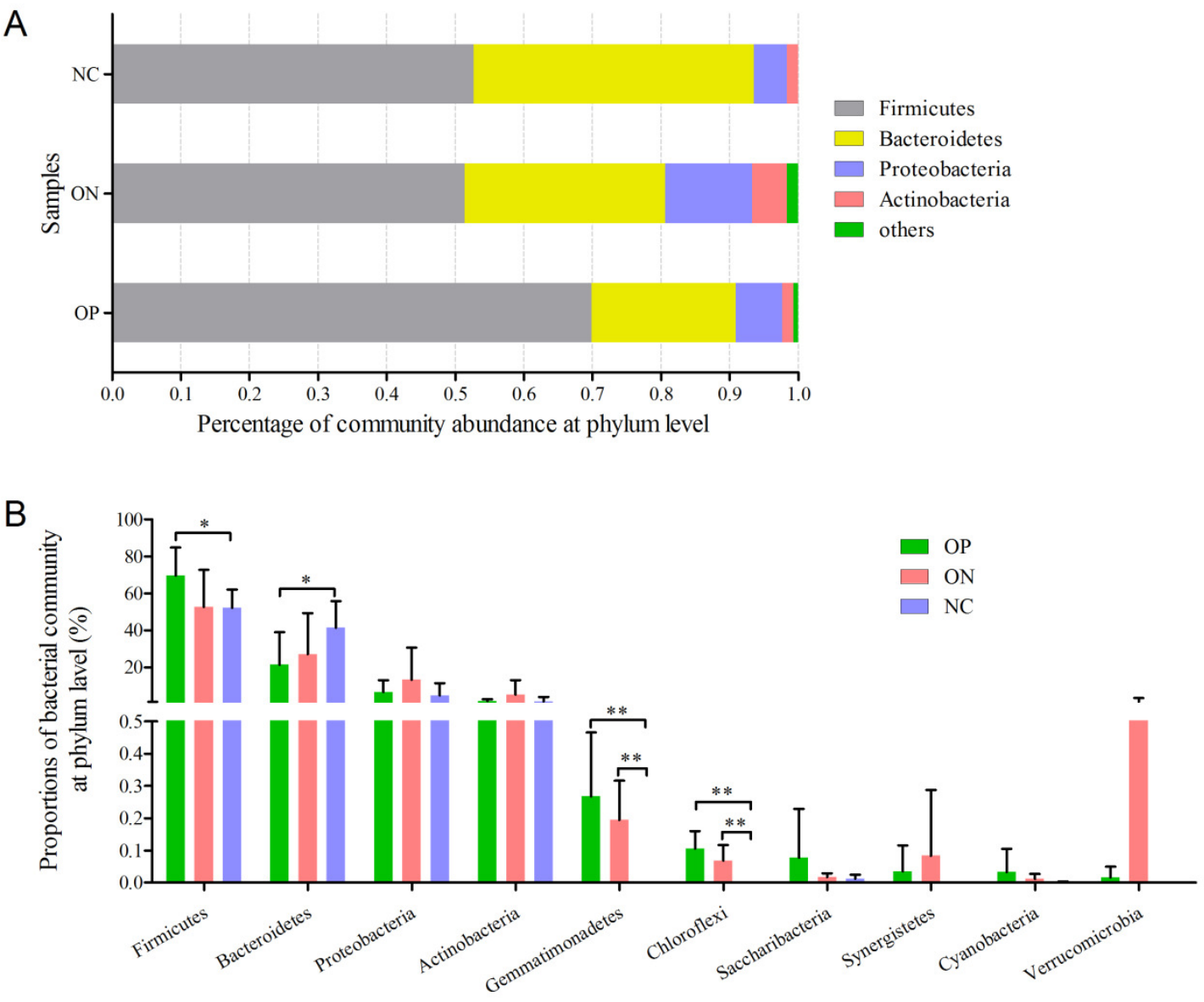
203

204

205

206

207

208

209

210

211

212

213

214

215

Figure 4. Bacterial community abundance at phylum level of each group. $(A)$ Bacterial community abundance barplot at phylum level. $(B)$ Significance of the top 10 bacterial community abundance at phylum level. ${ }^{*} 0.01<\mathrm{p} \leq 0.05, * * 0.001<\mathrm{p} \leq 0.01$ based on Mann-Whitney U-test.

At the genus level, a total of 21 genera with proportions above $1 \%$ were detected, as visualized in Figure 5. Bacteroides accounted for the largest proportion in all samples. In the NC group, 3 genera (Bacteroides, Faecalibacterium and Prevotella) contributed more than half of the bacterial community. In the ON and OP groups, 5 and 11 genera, respectively, accounted for $50 \%$ of the bacterial community. Differentiation analysis of the 21 genera is presented in Figure 5B. The Blautia, Parabacteroides and Ruminococcaceae genera differed significantly between the OP and NC groups. Figure S3 depicts the collinearity diagram for the bacterial community and samples from all three groups.
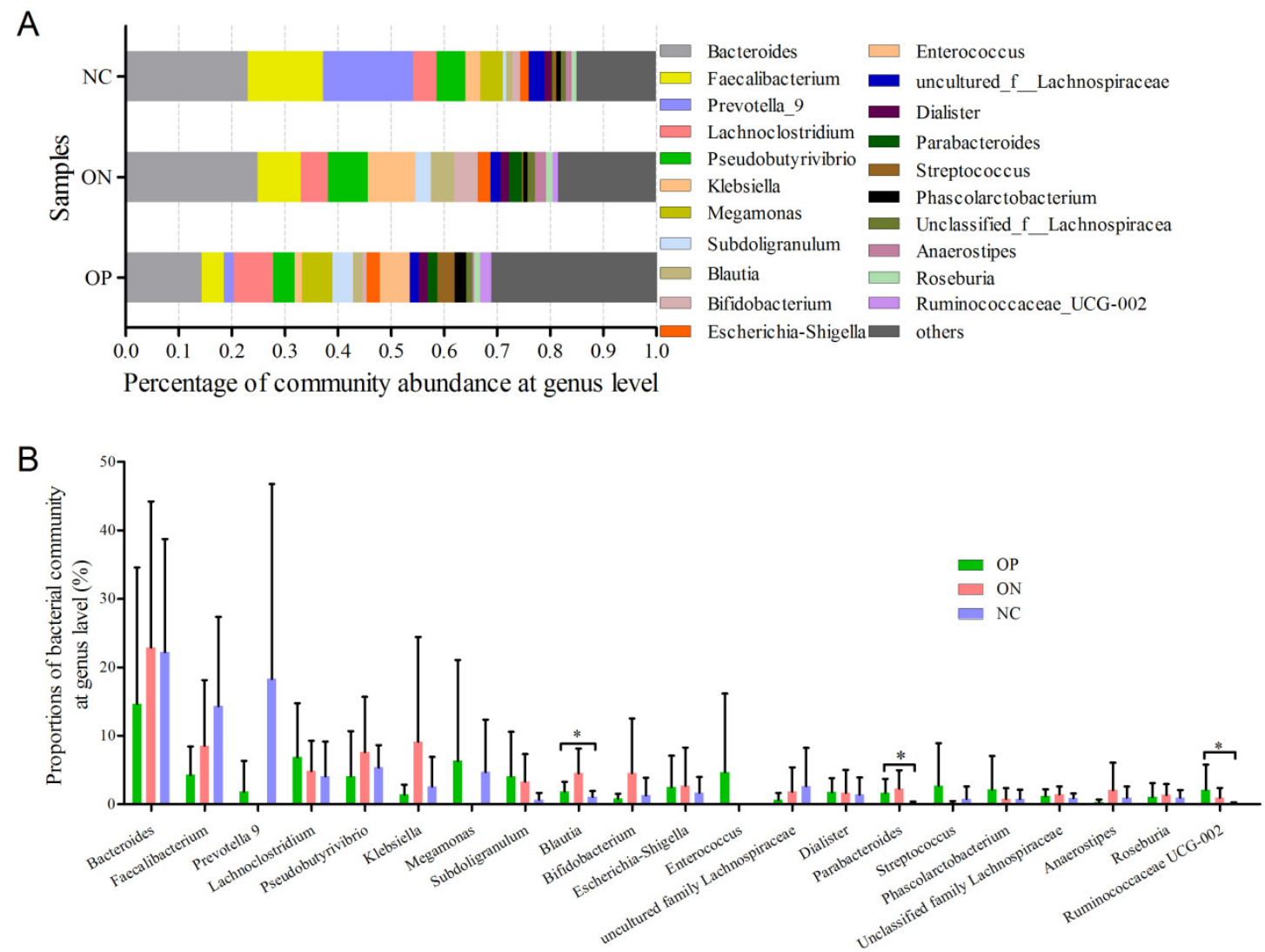

216

Figure 5. Bacterial community abundance at genus level of each group. (A) Bacterial community abundance barplot at genus level. $(B)$ Significance of the 10 bacterial community abundance at genus level.

$* 0.01<\mathrm{p} \leq 0.05$ based on Mann-Whitney U-test.

We further applied linear discriminant analysis (LDA) combined effect size measurements (LEfSe) to explore the significant changes and relative richness of the bacterial community in the $\mathrm{OP}, \mathrm{ON}$, and NC groups, at phylum and genus levels. Figure 6 summarizes the enrichment and 
224

225

226

227

228

229

230

variations in bacterial community for all three groups. At the phylum level, 1 phylum and 7 phylum communities were enriched in the $\mathrm{ON}$ and $\mathrm{OP}$ group, respectively, while no community in the NC group was enriched. At the genus level, 35 genus, 5 genus and 2 genus communities were enriched in the $\mathrm{OP}, \mathrm{ON}$ and $\mathrm{NC}$ groups, respectively. The significance and variance of bacterial communities, as determined by sequencing analysis, may help discriminate OP or ON patients from NC subjects.
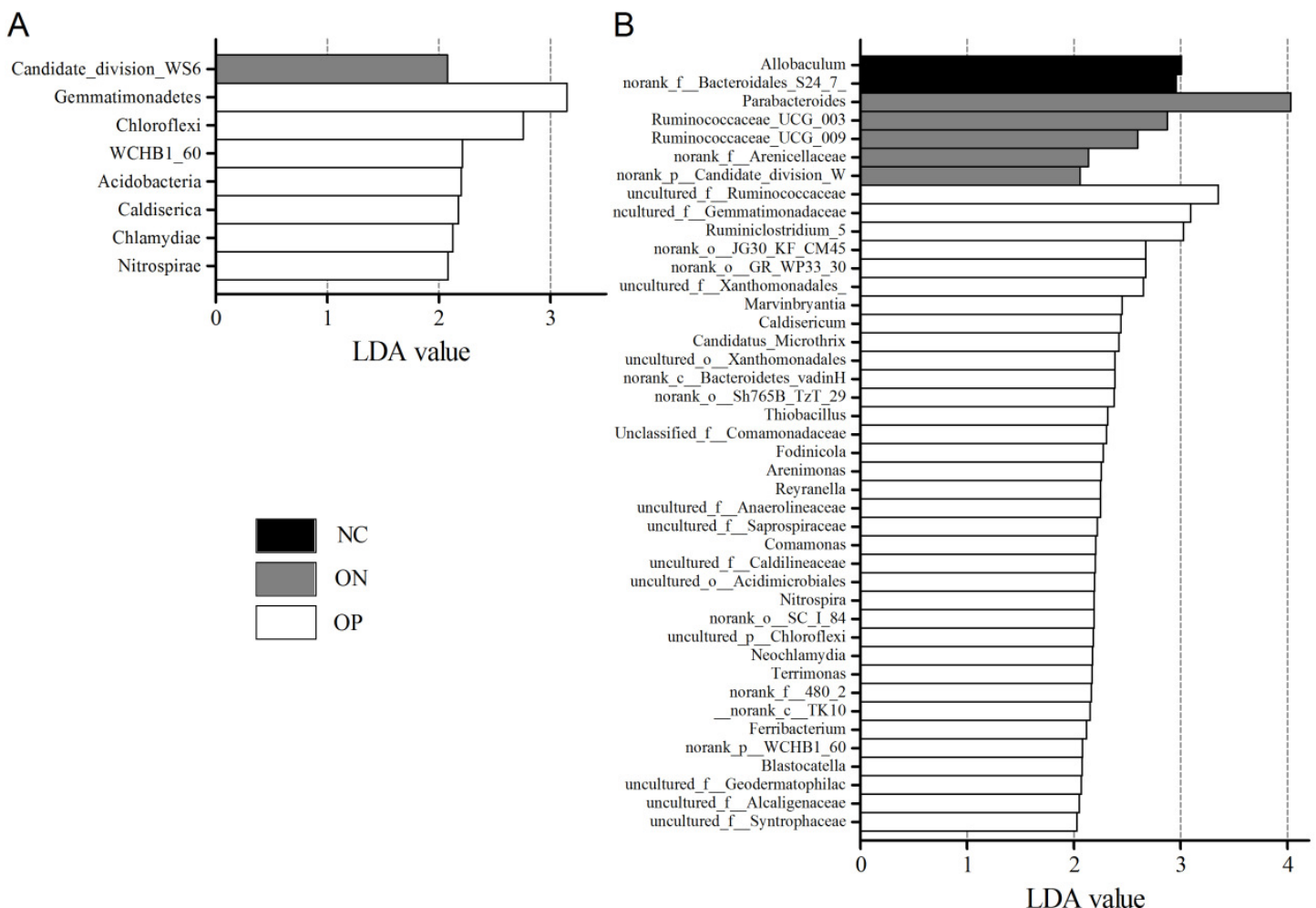

Figure 6. LEfSe at the phylum and genus level of each group. (A) LEfSe bar at phylum level. (B) LEfSe bar at genus level. $\mathrm{P}<0.05$, LDA value $>2$.

\section{DISCUSSION}

The human microbiome, referred to as our second genome, can influence genetic diversity, immunity and metabolism (Grice \& Segre 2012; Solt et al. 2011). All of the bacteria in specific samples can now be detected based on microbiota DNA sequencing. Research focused on gut microbiota and bone metabolism has recently emerged. Our study is the first survey about composition and diversity analysis of gut microbiota in osteoporosis, osteopenia patients and healthy controls using metagenomic sequencing. The results indicate that bacterial component structure and diversity are altered in osteoporosis and osteopenia patients as compared with normal controls, this supported the perspective that the bone health can be affected by the gut microbiota.

Microbiota diversity analysis is valuable for quantifying the bacterial component and relative richness of a specific community. Our investigation of alpha diversity revealed an 
247

248

249

250

251

252

253

254

255

256

257

258

259

260

261

262

263

264

265

266

267

268

269

270

271

272

273

274

275

276

277

278

279

280

281

282

283

284

285

286

287

elevation of diversity estimators in the OP and ON groups. Hierarchical clustering and PCoA analysis of beta diversity was able to discriminate the NC group from the OP and ON groups. These results suggested that a rich diversity of gut microbiota may be related to the reduction of bone mass. In OP group, the proportion of Firmicutes phyla increased and the proportion of Bacteroidetes decreased significantly $(p<0.05)$ compared with that in NC group. Several communities present at low levels in the OP and ON groups were absent in the NC group (e.g., Gemmatimonadetes Chloroflexi and Synergistetes). At the genus level, 21 genera with proportions over 1\% were identified. Bacteroides, Faecalibacterium and Prevotella were the top 3 genera in the NC group, while Prevotella was not observed in the $\mathrm{ON}$ and was present at low levels in the OP group. The Lachnoclostridium and Klebsiella genera were more abundant in the $\mathrm{OP}$ and $\mathrm{ON}$ groups as compared to the $\mathrm{NC}$ group. We further identified the enriched and significant community in each group and speculated that these communities may be considered as specific biomarkers for the reduction of bone mass.

The underlying mechanisms of gut microbiota changes in osteoporosis and osteopenia patients remained to be explained. We hypothesized that the immune-inflammatory axis may act as the key bridge joining the gut microbiota to bone metabolism. Studies have shown that bone mass increased in germ-free (GF) mice compared with conventionally raised mice. The authors reported fewer osteoclasts, osteoclast precursor cells, CD4 (+) cells and inflammatory cytokines in the bone and bone marrow of GF mice. They also reported that bone mass could be normalized after gut microbiota transplantation in GF mice (Sjogren et al. 2012). Moreover, certain pre- and probiotics have been shown to increase bone mass (Bindels et al. 2015; Maekawa \& Hajishengallis 2014; Scholz-Ahrens et al. 2007). Research suggests that gut microbiota and specific probiotics may regulate IGF-1, TNF- $\alpha$ and IL-1 $\beta$, resulting in changes in bone formation and growth (Ohlsson et al. 2014; Yan et al. 2016).

Notably, this study does have certain limitations. The sample size may not have been large enough. The average age in the osteoporosis and osteopenia groups was 70 years, and the sex ratio of female : male is $5: 1$ in the two groups. The occurrence of osteoporosis is more common with age, and is more common in females than males. It was reported that the osteoporosis prevalence ranged from $9 \%$ to $38 \%$ for females and $1 \%$ to $8 \%$ for males in different countries (Wade et al. 2014). In this study, the subjects in OP and ON groups were chosen randomly according with the recruiting criteria, and we further recruited the normal controls also at the same age and sex ratio to keep a balance. In view of this, we should consider the relevant hormonal changes, with corresponding effects on bone metabolism, because postmenopausal women are at high risk for osteoporosis (Cappola \& Shoback 2016). Researchers have reported that prebiotics improve calcium absorption, calcium accretion in bone and BMD in adolescents as well as postmenopausal female subjects (Roberfroid et al. 2010). Thus, dietary intake (e.g., pre- or probiotics) may alter bone metabolism in both pre- and post-menopausal women.

According to recent reports, studies in microbiota research have increased, which focusing on exploring new approaches for disease diagnosis and treatment (Castro-Nallar et al. 2015; Vernocchi et al. 2016). In our research, we explored gut microbiota diversity changes in primary osteoporosis and osteopenia patients. Further studies are required to understand the gut 
288

289

290

291

292

293

294

295

296

297

298

299

300

301

302

303

304

305

306

307

308

309

310

311

312

313

314

315

316

317

318

319

320

321

322

323

324

325

326

327

328 microbiota as a regulator for bone mass and evaluate it as a novel biomarker for osteoporosis

\section{Acknowledgments}

We would like to thank Kuan Liu, Sales Engineer from Majorbio, Shanghai, for the technology guidance.

\section{References}

Bindels LB, Delzenne NM, Cani PD, and Walter J. 2015. Towards a more comprehensive concept for prebiotics. Nat Rev Gastroenterol Hepatol 12:303-310.

Caporaso JG, Kuczynski J, Stombaugh J, Bittinger K, Bushman FD, Costello EK, Fierer N, Pena AG, Goodrich JK, Gordon JI, Huttley GA, Kelley ST, Knights D, Koenig JE, Ley RE, Lozupone CA, McDonald D, Muegge BD, Pirrung M, Reeder J, Sevinsky JR, Turnbaugh PJ, Walters WA, Widmann J, Yatsunenko T, Zaneveld J, and Knight R. 2010. QIIME allows analysis of high-throughput community sequencing data. Nat Methods 7:335-336.

Cappola AR, and Shoback DM. 2016. Osteoporosis Therapy in Postmenopausal Women With High Risk of Fracture. Jama 316:715-716.

Castro-Nallar E, Bendall ML, Perez-Losada M, Sabuncyan S, Severance EG, Dickerson FB, Schroeder JR, Yolken RH, and Crandall KA. 2015. Composition, taxonomy and functional diversity of the oropharynx microbiome in individuals with schizophrenia and controls. PeerJ 3:e1140.

Chung HJ, Cho L, Shin JS, Lee J, Ha IH, Park HJ, and Lee SK. 2014. Effects of JSOG-6 on protection against bone loss in ovariectomized mice through regulation of osteoblast differentiation and osteoclast formation. $B M C$ Complement Altern Med 14:184.

Edgar RC. 2013. UPARSE: highly accurate OTU sequences from microbial amplicon reads. Nat Methods 10:996-998. Ejtahed HS, Soroush AR, Angoorani P, Larijani B, and Hasani-Ranjbar S. 2016. Gut Microbiota as a Target in the Pathogenesis of Metabolic Disorders: A New Approach to Novel Therapeutic Agents. Horm Metab Res 48:349-358.

Grice EA, and Segre JA. 2012. The human microbiome: our second genome. Annu Rev Genomics Hum Genet 13:151-170.

Harada S, and Rodan GA. 2003. Control of osteoblast function and regulation of bone mass. Nature 423:349-355.

Jiang X, and Takacs-Vesbach CD. 2017. Microbial community analysis of pH 4 thermal springs in Yellowstone National Park. Extremophiles 21:135-152. 10.1007/s00792-016-0889-8.

Lauber CL, Hamady M, Knight R, and Fierer N. 2009. Pyrosequencing-based assessment of soil pH as a predictor of soil bacterial community structure at the continental scale. Applied and Environmental Microbiology 75:5111-5120. 10.1128/aem.00335-09.

Li H, Li T, Beasley DE, Hedenec P, Xiao Z, Zhang S, Li J, Lin Q, and Li X. 2016a. Diet Diversity Is Associated with Beta but not Alpha Diversity of Pika Gut Microbiota. Front Microbiol 7:1169.

Li H, Qu J, Li T, Li J, Lin Q, and Li X. 2016b. Pika Population Density Is Associated with the Composition and Diversity of Gut Microbiota. Front Microbiol 7:758.

Lu YC, Lin YC, Lin YK, Liu YJ, Chang KH, Chieng PU, and Chan WP. 2016. Prevalence of Osteoporosis and Low Bone Mass in Older Chinese Population Based on Bone Mineral Density at Multiple Skeletal Sites. Sci Rep 6:25206. 
329

330

331

332

333

334

335

336

337

338

339

340

341

342

343

344

345

346

347

348

349

350

351

352

353

354

355

356

357

358

359

360

361

362

363

364

365

366

367

368

369

Maekawa T, and Hajishengallis G. 2014. Topical treatment with probiotic Lactobacillus brevis CD2 inhibits experimental periodontal inflammation and bone loss. J Periodontal Res 49:785-791.

McCabe L, Britton RA, and Parameswaran N. 2015. Prebiotic and Probiotic Regulation of Bone Health: Role of the Intestine and its Microbiome. Curr Osteoporos Rep 13:363-371.

Ohlsson C, Engdahl C, Fak F, Andersson A, Windahl SH, Farman HH, Moverare-Skrtic S, Islander U, and Sjogren K. 2014. Probiotics protect mice from ovariectomy-induced cortical bone loss. PLoS One 9:e92368.

Perry GH, Marioni JC, Melsted P, and Gilad Y. 2010. Genomic-scale capture and sequencing of endogenous DNA from feces. Mol Ecol 19:5332-5344.

Pollitzer WS, and Anderson JJ. 1989. Ethnic and genetic differences in bone mass: a review with a hereditary vs environmental perspective. Am J Clin Nutr 50:1244-1259.

Quast C, Pruesse E, Yilmaz P, Gerken J, Schweer T, Yarza P, Peplies J, and Glockner FO. 2013. The SILVA ribosomal RNA gene database project: improved data processing and web-based tools. Nucleic Acids Res 41:D590596.

Roberfroid M, Gibson GR, Hoyles L, McCartney AL, Rastall R, Rowland I, Wolvers D, Watzl B, Szajewska H, Stahl B, Guarner F, Respondek F, Whelan K, Coxam V, Davicco MJ, Leotoing L, Wittrant Y, Delzenne NM, Cani PD, Neyrinck AM, and Meheust A. 2010. Prebiotic effects: metabolic and health benefits. Br J Nutr 104 Suppl 2:S1-63.

Scholz-Ahrens KE, Ade P, Marten B, Weber P, Timm W, Acil Y, Gluer CC, and Schrezenmeir J. 2007. Prebiotics, probiotics, and synbiotics affect mineral absorption, bone mineral content, and bone structure. J Nutr 137:838s-846s.

Segata N, Izard J, Waldron L, Gevers D, Miropolsky L, Garrett WS, Huttenhower C. 2011. Metagenomic biomarker discovery and explanation. Genome Biol 12:R60.

Sjogren K, Engdahl C, Henning P, Lerner UH, Tremaroli V, Lagerquist MK, Backhed F, and Ohlsson C. 2012. The gut microbiota regulates bone mass in mice. J Bone Miner Res 27:1357-1367.

Solt I, Kim MJ, and Offer C. 2011. [The human microbiome]. Harefuah 150:484-488.

Steves CJ, Bird S, Williams FM, and Spector TD. 2016. The Microbiome and Musculoskeletal Conditions of Aging: A Review of Evidence for Impact and Potential Therapeutics. J Bone Miner Res 31:261-269.

Szafranski SP, Wos-Oxley ML, Vilchez-Vargas R, Jauregui R, Plumeier I, Klawonn F, Tomasch J, Meisinger C, Kuhnisch J, Sztajer H, Pieper DH, and Wagner-Dobler I. 2015. High-resolution taxonomic profiling of the subgingival microbiome for biomarker discovery and periodontitis diagnosis. Appl Environ Microbiol 81:1047-1058.

Van Horn DJ, Wolf CR, Colman DR, Jiang X, Kohler TJ, McKnight DM, Stanish LF, Yazzie T, and Takacs-Vesbach CD. 2016. Patterns of bacterial biodiversity in the glacial meltwater streams of the McMurdo Dry Valleys, Antarctica. FEMS Microbiol Ecol 92. 10.1093/femsec/fiw148.

Vernocchi P, Del Chierico F, and Putignani L. 2016. Gut Microbiota Profiling: Metabolomics Based Approach to Unravel Compounds Affecting Human Health. Front Microbiol 7:1144.

Wade SW, Strader C, Fitzpatrick LA, Anthony MS, O'Malley CD. 2014. Estimating prevalence of osteoporosis: examples from industrialized countries. Arch Osteoporos 9:182.

Wang Q, Garrity GM, Tiedje JM, and Cole JR. 2007. Naive Bayesian classifier for rapid assignment of rRNA sequences into the new bacterial taxonomy. Appl Environ Microbiol 73:5261-5267.

Weaver CM. 2015. Diet, gut microbiome, and bone health. Curr Osteoporos Rep 13:125-130.

Yan J, Herzog JW, Tsang K, Brennan CA, Bower MA, Garrett WS, Sartor BR, Aliprantis AO, and Charles JF. 2016. Gut

PeerJ reviewing PDF | (2017:03:17027:2:0:NEW 10 May 2017) 

e7563. 